\title{
The Assessment of the Effect of the Haemovigilance Education by the Conceptual Map on the Knowledge of Nursing Students
}

\section{Mohammad Saeed Mirzaee (MSc) $)^{1, *}$, Mahmood Karimi (PhD) ${ }^{2}$}

${ }^{I}$ Faculty Member, School of Nursing, Saveh University of Medical Sciences, Saveh, Iran

${ }^{2}$ Assistant Professor of Health Education and Promotion, Social Determinants of Health Research Center, Saveh University of Medical Sciences, Saveh, Iran

* Corresponding Author: Mohammad Saeed Mirzaee, Social Determinants of Health Research Center, Saveh University of Medical Sciences, Saveh, Iran. Email: saeedmirzaee75@gmail.com

\section{Abstract}

Received: $12 / 06 / 2018$

Accepted: 03/08/2018

\section{How to Cite this Article:}

Mirzaee MS, Karimi M. The

Assessment of the Effect of the Haemovigilance Education by the Conceptual Map on the Knowledge of Nursing Students. Pajouhan Scientific Journal. 2018; 17(1): 51-56. DOI: 10.29252/psj.17.1.51
Background and Objective: Haemovigilance recognizes and eliminates complications caused by blood transfusions, improves the quality of nursing care, and increases patient satisfaction. With regards to the importance of the problem, this study aimed to determine the effect of haemovigilance education, using a conceptual map on the knowledge of nursing students.

Materials and Methods: The present study was a semi-experimental study carried out at the Saveh University of Medical Science in 2017. Samples consisted of 60 nursing students of the Saveh University of Medical Science. They were selected by census sampling and based on entry and exit criteria and randomly assigned to two groups of test and control. For data collection use of the haemovigilance knowledge questionnaire. Its validity was confirmed and its reliability was reported as 0.83 using Cronbach's alpha coefficient. The training program was conducted in 4 sessions. For data analysis, descriptive statistics and inferential statistics (independent t-test, chi-square, Fisher's exact test and Wilcoxon test) and SPSS-25 software were used.

Results: There was a significant difference between the two groups in the test and control groups $(\mathrm{P}=0.0001)$. There was a significant difference in the level of knowledge among students between the two groups after intervention $(\mathrm{P}=0.0001)$. The students' knowledge score in the test and control groups was significantly different after the intervention $(\mathrm{P}=$ 0.0001). The mean change in knowledge of students before the intervention was significantly different in the test group $(\mathrm{P}=0.0001)$.

Conclusion: The Conceptual map is a new method that haemovigilance education through it increases the awareness of nursing students and reduces the complications of improper use of blood products in patients.

Keywords: Conceptual Map; Haemovigilance; Knowledge 


\section{محمد سعيد ميرزايى ا“*، محمود كريمى}

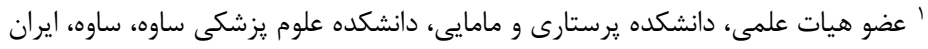

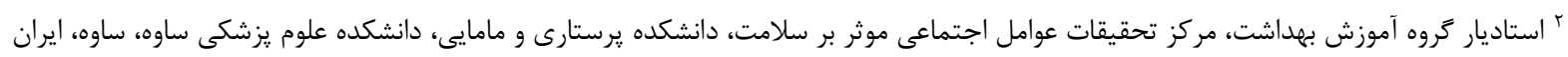
* نويسنده مسئول: محمد سعيد ميرزايى، دانشكده ير ستارى و مامايى، دانشكده علوم يزشكى ساوه، ساوه، ايران. ايميل: saeedmirzaee75@gmail.com

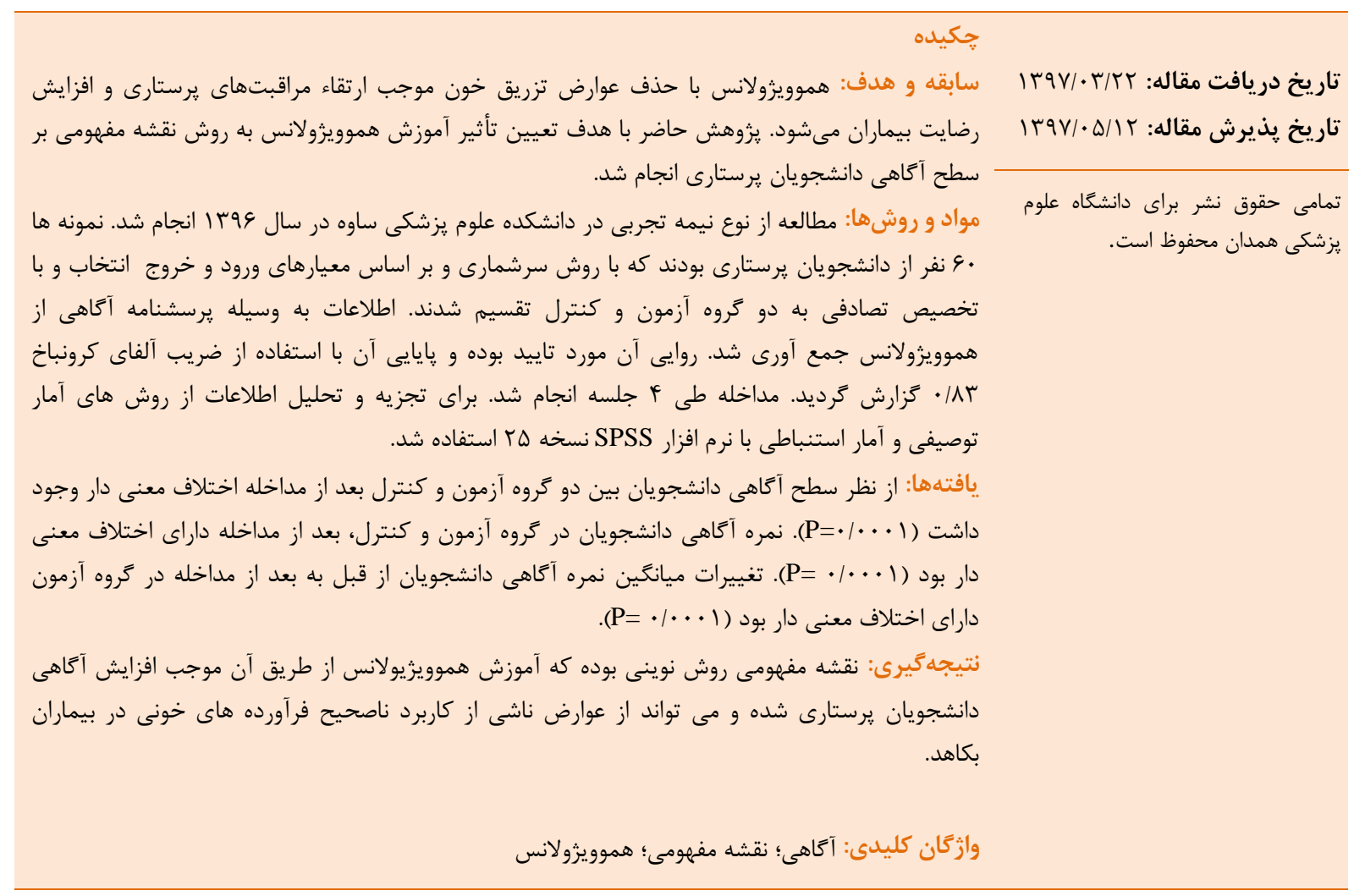

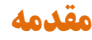

داشته باشند [؟]، شناخت عوارض ناتمانى ناشى از تزريق

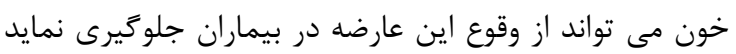

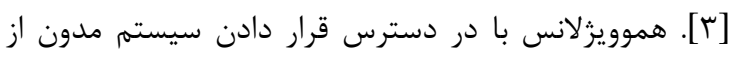

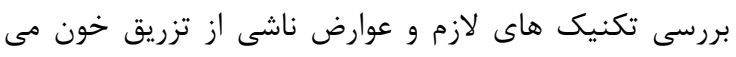

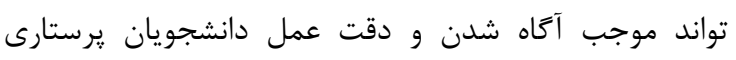

شود [F]

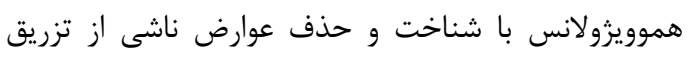

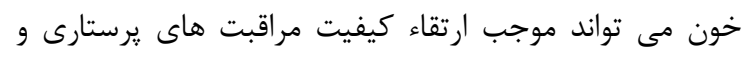

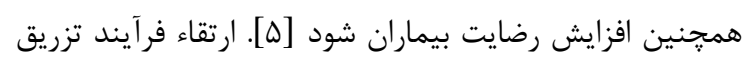

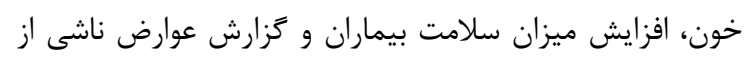

هموويزولانس شامل مجموعه اقداماتى زنجيره انتقال

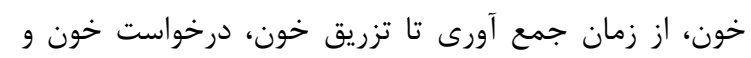

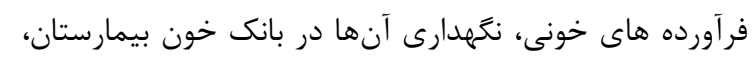

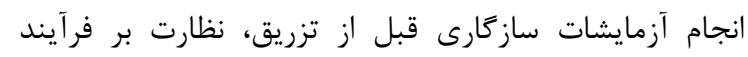

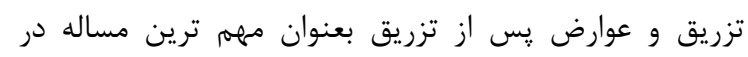

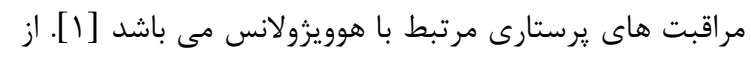

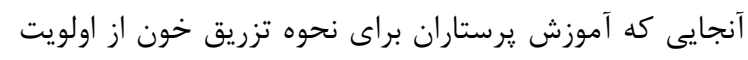

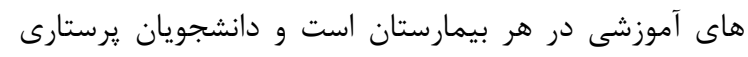

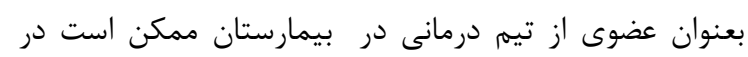

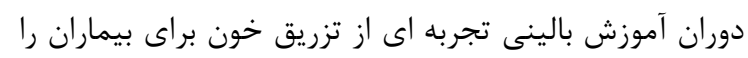


شماره IR.SAVEHUMS.REC1396.40 و كسب اجازه از مسئولين، به منظور جمع آورى اطلاعات از يرسشنامه آكاهى آنى

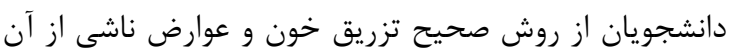

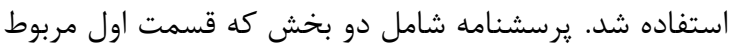

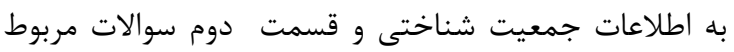
به آكاهى از روش صحيح تزريق خون مى باشد. •r سوال

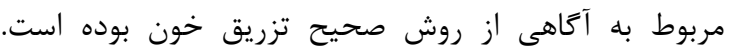

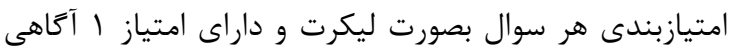

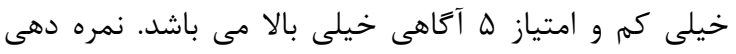

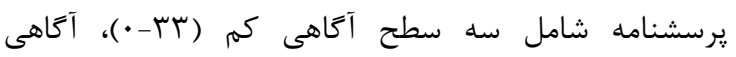

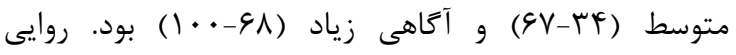

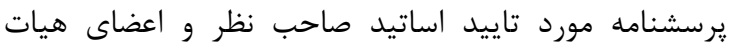

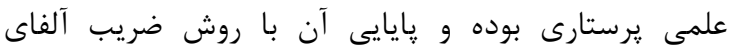

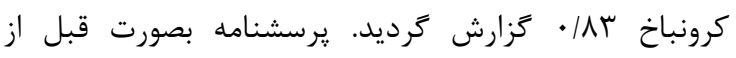

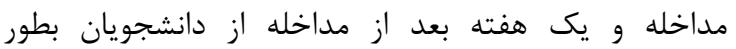

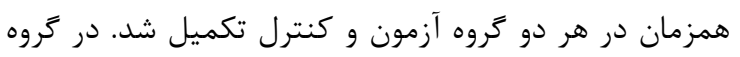

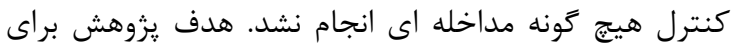

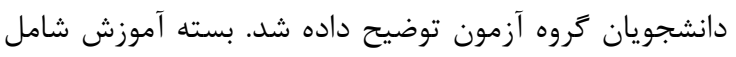

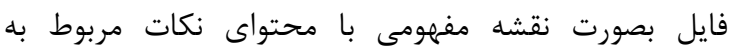

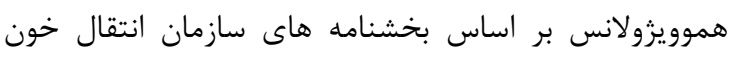

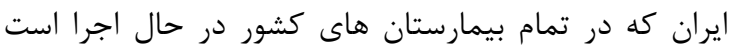

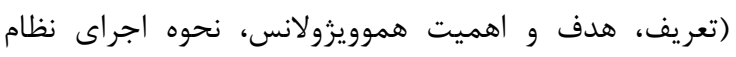

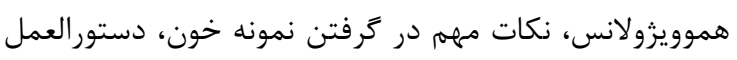

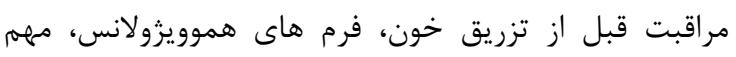

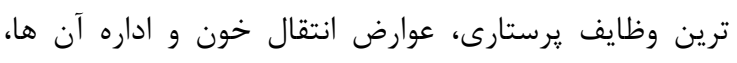

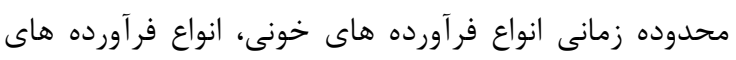

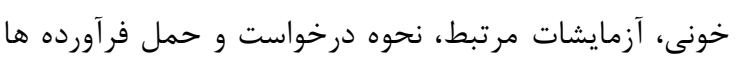

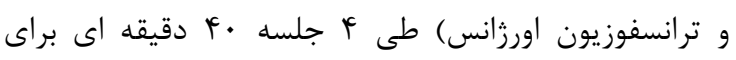

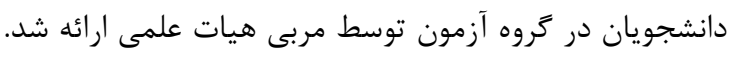

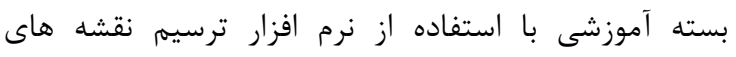

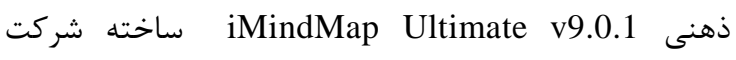
Tony Buzan Online

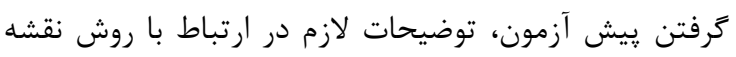

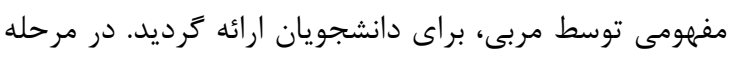

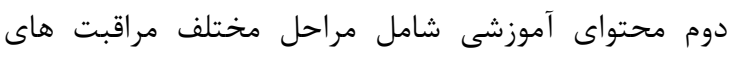

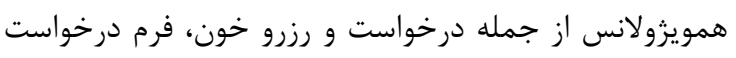

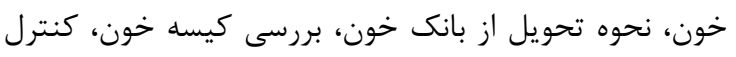

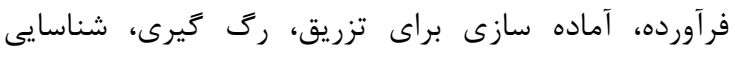

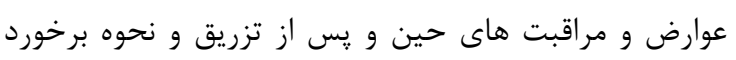

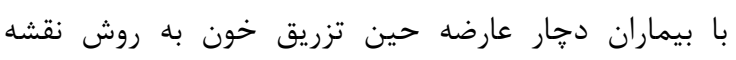

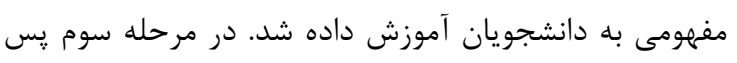

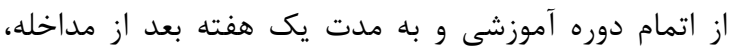

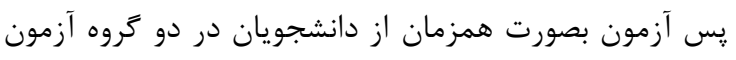

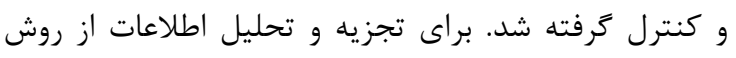

تزريق خون از جمله اهداف هموويزولانس است [ع] و در در أنقاز

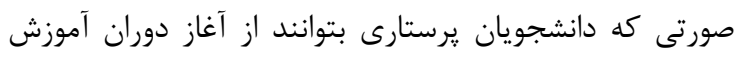

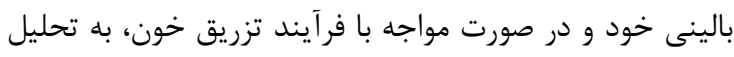

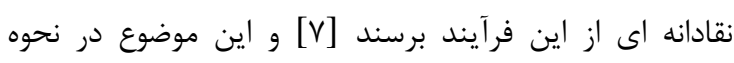

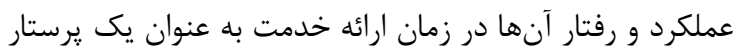

اثر كذار باشد [1]].

كسب آكاهى بعنوان شناخت و دركى مسايل مى تواند

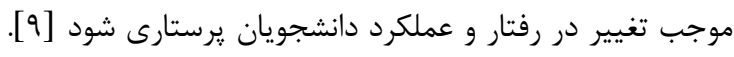

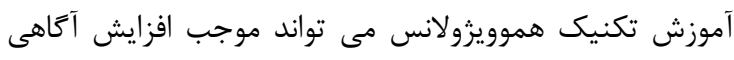

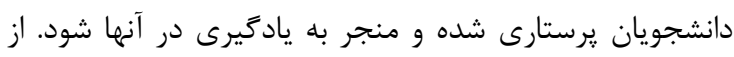

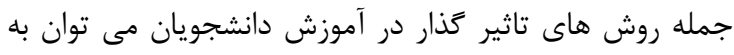

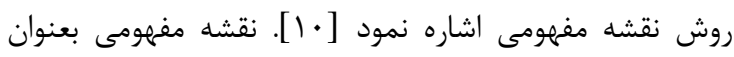

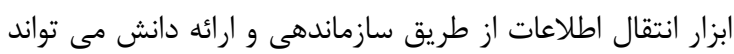

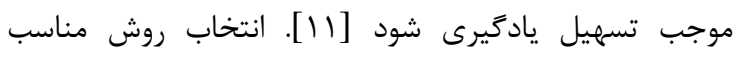

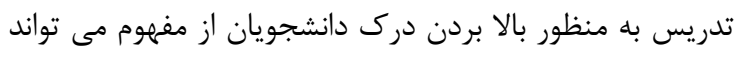

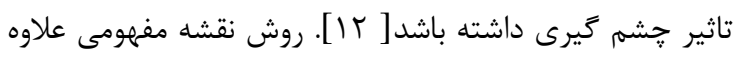

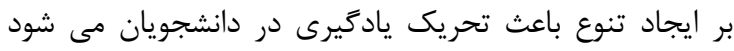

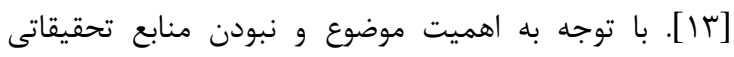

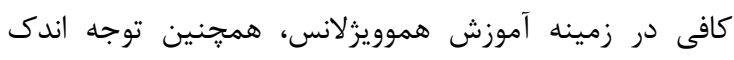

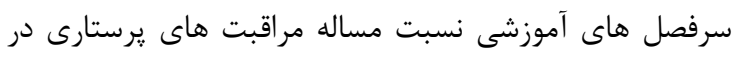

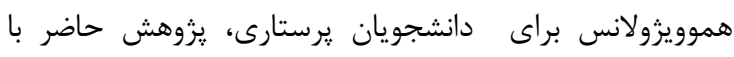

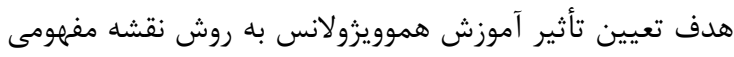

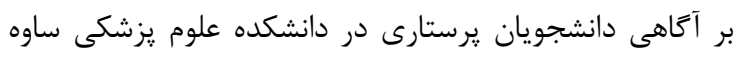

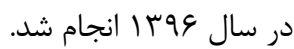

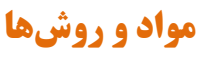

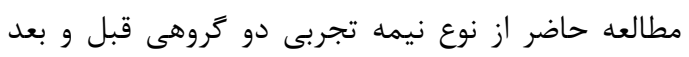

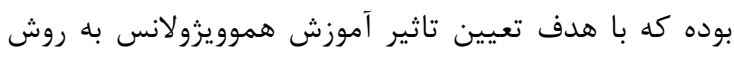

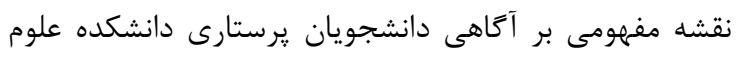

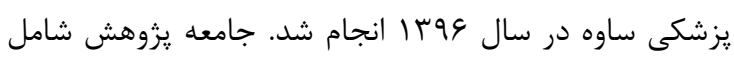

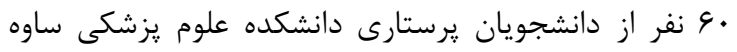

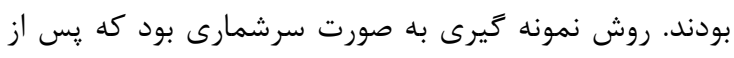

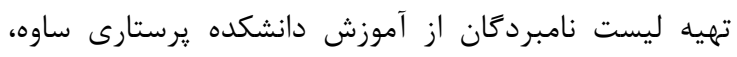

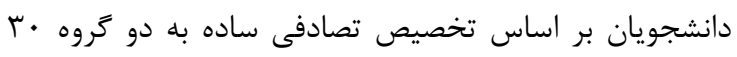

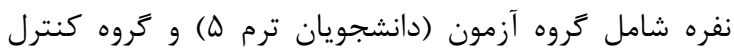

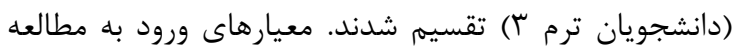

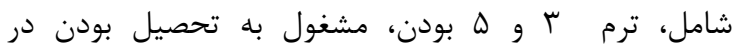

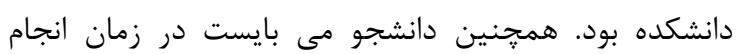

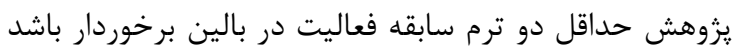

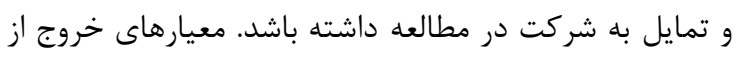

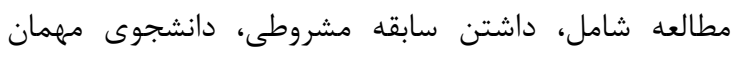

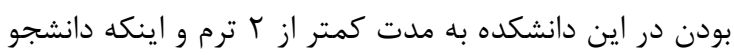

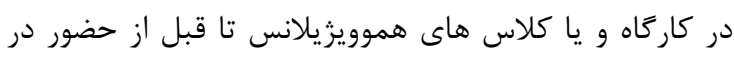

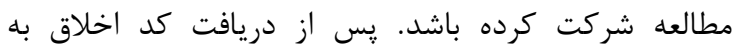


درصد دانشجويان در كروه آزمون داراى سطح آكاهى بالا و در

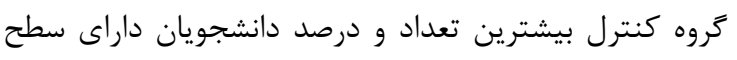

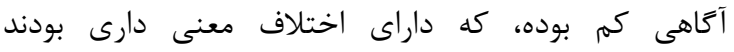

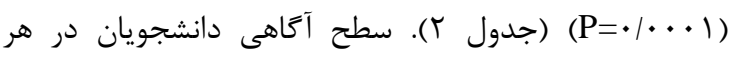

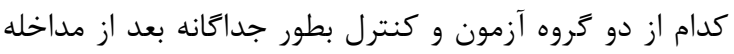

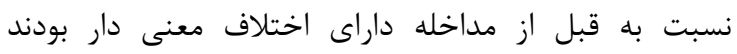

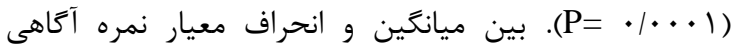

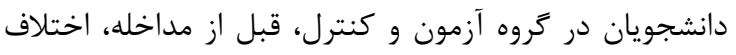

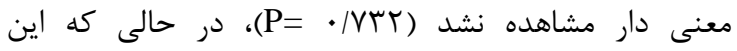

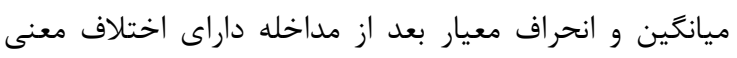

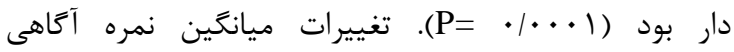

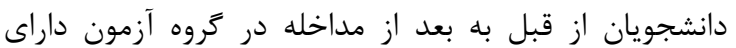

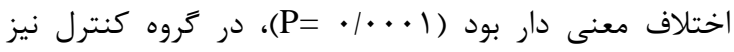

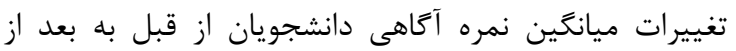

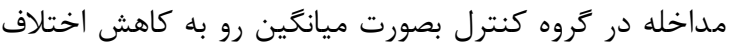

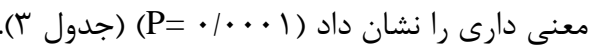

هاى آمارتوصيفى و آمار استنباطى شامل؛ فيشر، كاى دو،

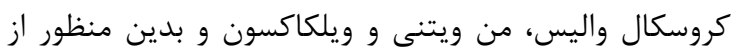
نرم افزار SPSS نسخه هـ استفاده شد.

\section{يافته ها}

ميانكَين و انحراف معيار سن در كروه مداخله و در كروه كنترل اختلاف معنى دارى نبوده است (P= (PA

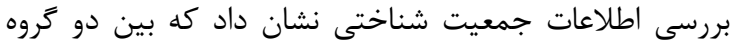

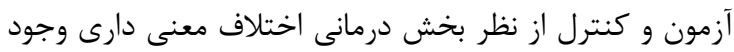

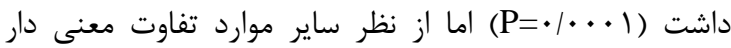

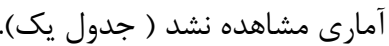

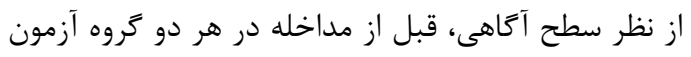

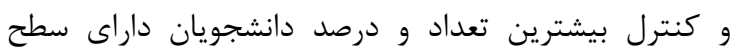

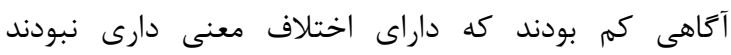
(P=•r/V)

جدول ا: تعيين تعداد و درصد اطلاعات جمعيت شناختى دانشجويان ترستارى در گروه آزمون و كنترل

\begin{tabular}{|c|c|c|c|c|c|c|}
\hline \multirow{3}{*}{ P-value } & \multicolumn{4}{|c|}{ تروه } & \multirow{3}{*}{\multicolumn{2}{|c|}{ فاكتور جمعيت شناختى }} \\
\hline & \multicolumn{2}{|c|}{ كروه كنترل } & \multicolumn{2}{|c|}{ كروه آزمون } & & \\
\hline & درصد & تعداد & درصد & تعداد & & \\
\hline 苇券 & $F \& \mid 9 V$ & If & $\Delta 9 / 99$ & IV & مرد مرد & \\
\hline & Tr/T & 19 & Frmp & ir & زن - ان & . \\
\hline$\approx .1 \cdot r$. & $9 \cdot$ & TV & 9. & TV & 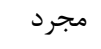 & \\
\hline & 1 . & r & 1 . & r & متاهل ل & \\
\hline & - & - & rब/99 & $\wedge$ & روان & \\
\hline & - & - & $r \cdot 1 \cdot 1$ & ir & اورزانس & \\
\hline 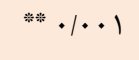 & - & - & אח/שr & 1 . & جراحى & بخش يرستارى \\
\hline & 4. & 11 & - & - & داخلى & \\
\hline & f. & ir & - & - & 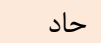 & \\
\hline
\end{tabular}

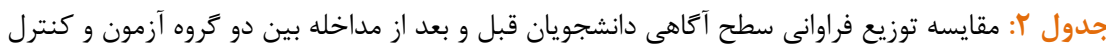

\begin{tabular}{|c|c|c|c|c|c|c|c|}
\hline \multirow{2}{*}{ P-value $*$} & \multicolumn{3}{|c|}{ كروه كنترل تعداد (درصد) } & \multicolumn{3}{|c|}{ كروه آزمون تعداد (درصد) } & \multirow[b]{2}{*}{ سطح آثاهى } \\
\hline & بالا & متوسط & هايين & بالا & متوسط & هايين & \\
\hline$\cdot$ / TIV & - & - & $r \cdot(1 \cdots)$ & - & I(T/Kr) & r৭ (१९|\&V) & قبل از مداخله \\
\hline$\cdot \mid \cdots \cdot 1$ & - & - & $r \cdot(1 \cdot \cdot)$ & $\operatorname{IV}(\Delta \& \mid \varphi \varphi)$ & $\|(F, T F)$ & - & بعد از مداخله \\
\hline
\end{tabular}

جدول بّ: ميانكين نمره آكاهى دانشجويان قبل از مداخله و بعد از مداخله بين دو گروه آزمون و كنترل

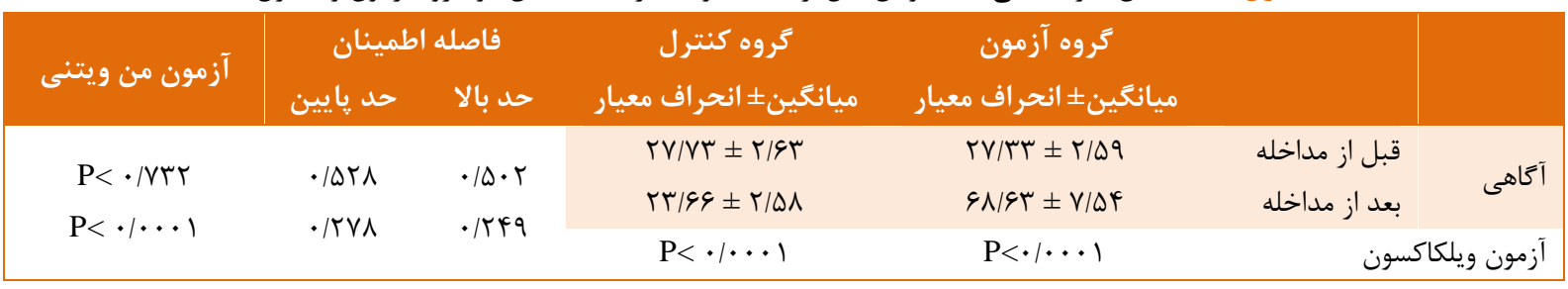


دانشجويان مشاهده نشد. فريكسو و همكاران در يزوهش خود

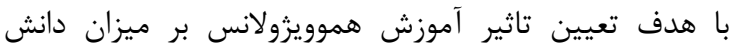

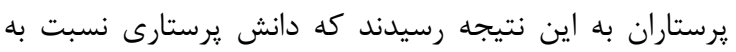
هموويزولانس در گروه آزمون قبل از مداخله يايين بوده و يس يس

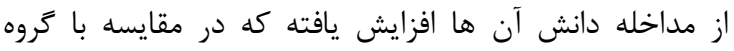

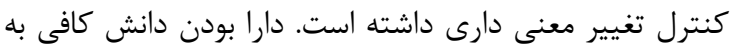

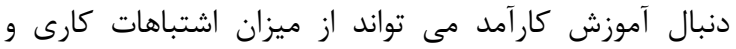

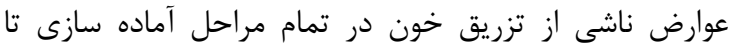

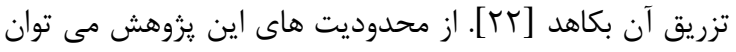

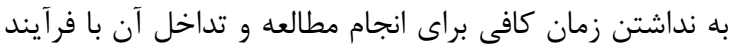
تحصيلى دانشجويان اشاره كرد كه با توجيه دانشويان و انجام بهام

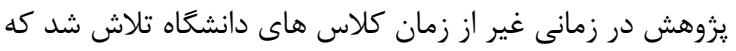
محدوديت ها كاهش يابند.

نتيجنه تَيرى با توجه به اينكه هر كونه اشتباه و آكاهى گايين كادر

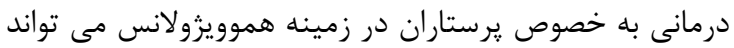

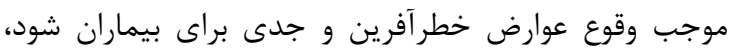

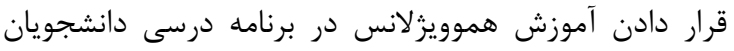

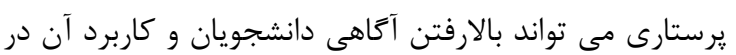

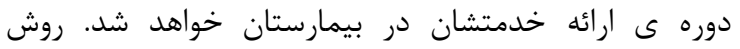

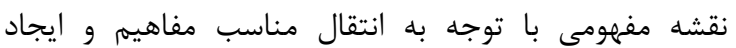

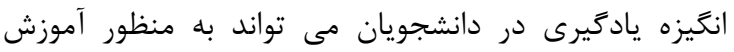

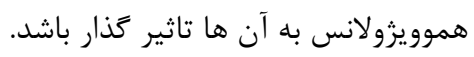

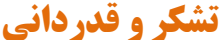

بدين وسيله از معاونت محترم يزوهشى دانشكده علوم

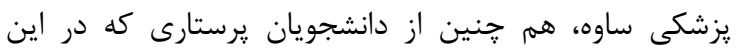
يروهش مشاركت داشتند كمال تشكر و قدردانى را داريم.

تضاد منافع اين مطالعه براى نويسند Fان هيج گونه تضاد منافعى نداشته

\section{REFERENCES}

1. C. Politis JC, Wiersum C, Richardson P, Robillard J, Jorgensen $\mathrm{P}$, et al. The international haemovigilance network database for the surveillance of adverse reactions and events in donors and recipients of blood components: technical issues and results. Vox sanguinis. 2016; 111.4: 409-417.

2. Shahshahani HJ. Status of Transfusion Medicine Education in Iran. Archives of Iranian Medicine. 2016; 19(6): 439-445.

3. Trudi G, Sue D, Matthew V, Linda C, Julie T. Patient blood management nurse vs transfusion nurse: is it time to merge? British Journal of Nursing. 2015; 24(9): 492-495.

4. Nair RB, Tejasree GR, R. Sirisha M, Shreya N, Neha P, et al. Assessment of Awareness on Haemovigilance among
آموزش يرستاران مى تواند در كاهش عوارض ناشى از

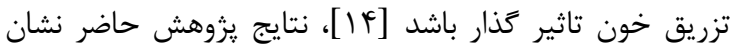

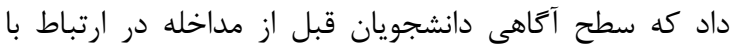

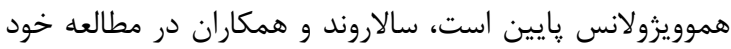

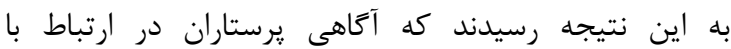

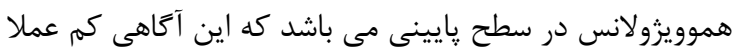

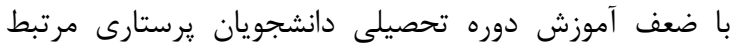

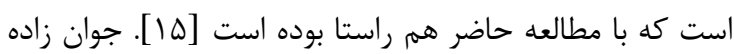

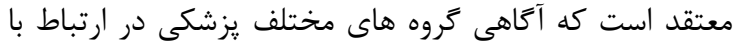

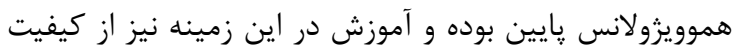

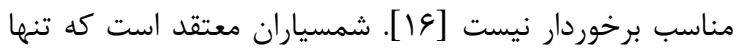

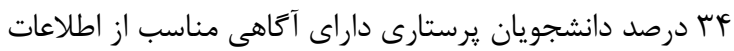

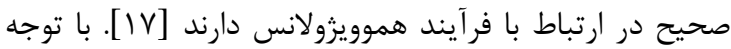

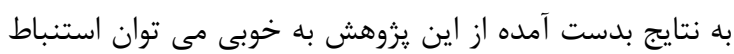

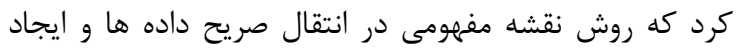

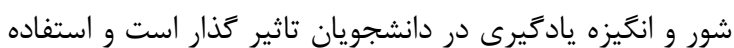

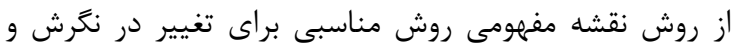

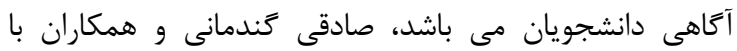
مقايسه تاثير آموزش نقشه مفهومى و سخنرانى بر خلى بلاقيت

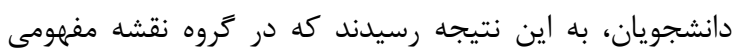

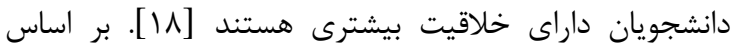

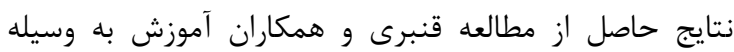
نقشه مفهومى باعث ارتقاء يادگيرى در دانشجويان در كروه

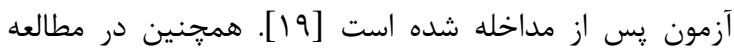
صادقى گَندمانى تدريس با روش نقشه مفهومى در گروه نقشه

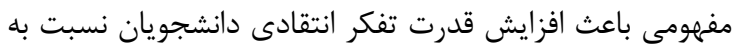

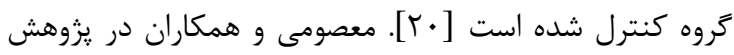

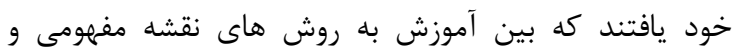

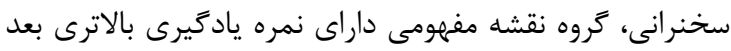

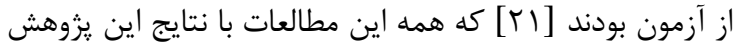

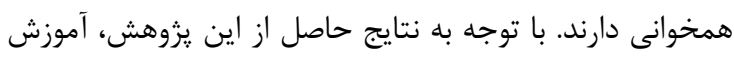

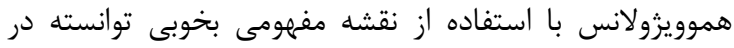

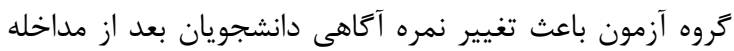

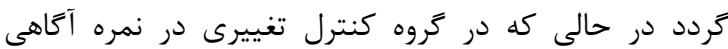

Health Care Professionals. Advanced Science Letters. 2017; 23(3):1777-1780.

5. Tajalli S, Nourian M, Rassouli M, Baghestani AR. Clinical Assessment of Nursing Care Regarding Hemovigilance in Neonatal Wards and Neonatal Intensive Care Units in Selected Hospitals Affiliated to Shahid Beheshti University of Medical Sciences (2013-2014). Iranian Red Crescent Medical Journal. 2015; 17(7): e19987

6. de Mel S, Lim S, Soekojo CY, Thow C, Lang SP, et al. Education-based interventions to minimize sampling errors in transfusion. ISBT Science Series. 2017; 12(2): 307-313.

7. Apphia JQT, Cindy CSL, Patrick YL, Simon C, Lydia STL, et al. Designing and evaluating the effectiveness of a serious 
game for safe administration of blood transfusion: A randomized controlled trial. Nurse education today. 2017; 55: 38-44.

8. Flood LS, Julie H. A comparative assessment of nursing students' cognitive knowledge of blood transfusion using lecture and simulation. Nurse education in practice. 2016; 16(1): 8-13.

9. Chinn P L, Maeona K K. Integrated Theory \& Knowledge Development in Nursing-E-Book. Elsevier Health Sciences, 2013.

10. Sharma U, Divya S, Kailash N K. Haemovigilance: a system to improve safety in blood transfusion process. World Journal of Pharmacy and Pharmaceutical Sciences. 2014; 3(6): 1889-98.

11. Jaafarpour M, Sanaz A, Mosayeb M. Does concept mapping enhance learning outcome of nursing students? Nurse education today. 2016; 36: 129-132.

12. Kaddoura M, VanDyke O, Cheng B, Shea-Foisy K. Impact of concept mapping on the development of clinical judgment skills in nursing students. Teaching and Learning in Nursing. 2016; 11(3): 101-107

13. Peter H, Ellinor E, Gita H, Albert W, Catharina SH. Group concept mapping for evaluation and development in nursing education. Nurse education in practice. 2016; 20: 147-153.

14. Mac Donald NE, O'Brien SF, Delage G. Transfusion and risk of infection in Canada: update 2012. Paediatrics \& child health. 2012; 17(10):102-6.

15. Salarvand S, Bahri N, Kiani N, Maafi M. Knowledge status of clinical care workers about safe transfusion and its related factors in academic hospitals in Gonabad University of Medical Sciences. Scientific Journal of Iranian Blood
Transfusion Organization. 2013; 10(1). 93-97

16. Marshall AL, Pruthi RK, Kreuter JD. Simulation-based Transfusion Education for Medical Students. Transfusion medicine reviews. 2018; 32(2): 123-124.

17. Shamshirian A, Alirahimi Z, Ghorbanpour A, Motamen S, Maadi N, et al. Knowledge and Awareness of Nursing Students on Blood Transfusion. International Journal of Medical Investigation. 2017; 6(4): 129-134.

18. Sadeghi-Gandomani H, Delaram M, Naseri-Brugeni N. Comparison of concept mapping and conventional teaching methods on creativity of nursing students. The Journal of Medical Education and Development. 2014; 9 (3) :48-57 [Persian]

19. Ghanbari A, Paryad E, Ehsani M. The Effectiveness of Conceptual Map Teaching Method on Short and Long Term Learning in Nursing Students. Strides in Development of Medical Education. 2011; 7(2) :112-118[Persian]

20. Hamidreza S, Sara S, Narges NB, Vahideh P. Comparison of the Effects of Concept Mapping and Conventional Teaching Methods on Critical Thinking of Nursing Students. Journal of Nursing Education. 2016; 5(4): 2733[Persian]

21. Masoumy M, Ebadi A, Raisifar A, hosseini R, Javanbakhtian R. Comparison of Two Teaching Methods on Nursing Students' Learning and Retention: Concept Mapping or Lecture? Iranian Journal of Medical Education. 2012; 12(7):498-507[Persian]

22. Freixo A, Matos I, Leite A, Silva A, Bischoff F, et al. Nurses knowledge in Transfusion Medicine in a Portuguese university hospital: the impact of an education. Blood Transfusion. 2017; 15(1): 49 